\title{
Analytical relations for the strain tensor components caused by acoustic waves with arbitrary wave vector directions in crystals: acousto-optic applications
}

\author{
Krupych O., Adamenko D. and Vlokh R. \\ Vlokh Institute of Physical Optics, 23 Dragomanov Street, 79005, Lviv, Ukraine
}

Received: 04.02.2019

\begin{abstract}
Analytical relations for the strain tensor components caused by acoustic waves with arbitrary wave vector directions in crystals are derived. Our approach involves standard rotation matrices and cross sections of the indicative surfaces of Christoffel tensor, which are made by the planes perpendicular to the wave vectors.
\end{abstract}

Keywords: acousto-optics, acoustic waves, strain tensor

PACS: $78.20 . \mathrm{Hp}, 62.65 .+\mathrm{k}, 62.20 . \mathrm{Fe}$

UDC: $534.231 .2+535.42$

\section{Introduction}

One of the major problems of acousto-optic (AO) materials science is searching for the crystals with high enough values of acousto-optic figure of merit (AOFM). This parameter plays a role of a coupling coefficient between the efficiency of AO interactions and the acoustic wave (AW) power. If the AOFM is high, the operating power can be reduced, thus implying decreased energy consumption, which is always important. The AOFM is defined as $M_{2}=n^{6} p_{e f}^{2} / \rho v^{3}$, where $n$ is the refractive index, $\rho$ the material density, $v$ the AW velocity, and $p_{e f}$ the effective elastooptic coefficient (EEC) [1]. Most of these constitutive coefficients are associated with tensor quantities and so depend on the geometry of AO interactions. Hence, one can, in principle, find an optimal phase-matched vector diagram, for which a maximal AOFM is reached, when choosing properly the geometry of AO diffraction.

The above problem is not as easy as it might have seemed at a first glance. In general, the AW velocities can be obtained following from the Christoffel tensor after finding its eigenvalues, which can be done either numerically or analytically. However, the EEC is determined by cumbersome relations that depend upon the directions of propagation and polarization of the interacting optical waves and the AW. Here the polarization and propagation directions of the AW determine nonzero strain tensor components, which affect optical impermeability tensor through elasto-optic effect, and specify the set of elasto-optic tensor components involved into the EEC. The strain tensor components are given by derivatives of the components of displacement vector with respect to the coordinates [2]. Notice that the displacement vectors for each of the three acoustic eigenwaves (one quasi-longitudinal and two quasi-transverse ones) coincide with the eigenvectors of the Christoffel tensor. However, accounting for the effect of non-orthogonality of the AWs leads to appearance of more than one off-diagonal components in the Christoffel tensor, thus making it impossible to solve the appropriate equation analytically. Nonetheless, it is necessary to obtain the analytical relations for the EEC if one has to grasp the reasons for certain diffraction efficiency.

Ukr. J. Phys. Opt. 2019, Volume 20, Issue 1 
As a consequence, for solving this problem one is usually forced to use the numerical approach $[3,4]$ which is not as informative as the analytical one. The other alternative approaches are based on further approximations. For example, the AOFM anisotropy in lithium niobate has been calculated in Ref. [5] via the analysis of only anisotropic AO interactions with the longitudinal AWs. Only a collinear type of AO interactions has been analyzed for the $\mathrm{LiNbO}_{3}$ crystals in the work [6]. In our recent works [7-9] we have neglected completely the nonorthogonality of AWs. As a matter of fact, such an approach can be justified only when the angle of non-orthogonality is small enough. The other possibilities appear if one restricts oneself to the AO interactions in the principal crystallographic planes only. Then one can indeed derive the analytical relations for the EEC with accounting for the polarization non-orthogonality for the AWs [10]. On the other hand, this simplified approach can be applied to the crystals of at least orthorhombic symmetry, because the general problem of analytical solutions reappears again for the crystalline materials of lower symmetries.

In the present work we develop a general approach in order to obtain analytical relations for the strain tensor components, which are caused by the AWs that propagate along arbitrary directions, with taking the non-orthogonality (quasi-longitudinality or quasi-transversality) effect into account.

\section{Derivation of analytical relations}

To describe all the possible directions of AW propagation, we start with the initial crystallophysical coordinate system $X Y Z$. This is a Cartesian system where all the constitutive tensors are represented. To pass to arbitrary directions in crystals, we need to rotate the system mentioned above around two non-collinear axes. To be specific, one can first rotate the coordinate system $X Y Z$ around the $Z$ axis by the angle $+\varphi$ (counter-clockwise), which gives the system $X^{\prime} Y^{\prime} Z^{\prime}$ with $Z^{\prime}=Z$ (see Fig. 1). The second rotation is performed around the $Y^{\prime}$ axis by the angle $+\theta$. In this manner we obtain the coordinate system $X^{\prime \prime} Y^{\prime \prime} Z^{\prime \prime}$, with $Y^{\prime \prime}=Y^{\prime}$.

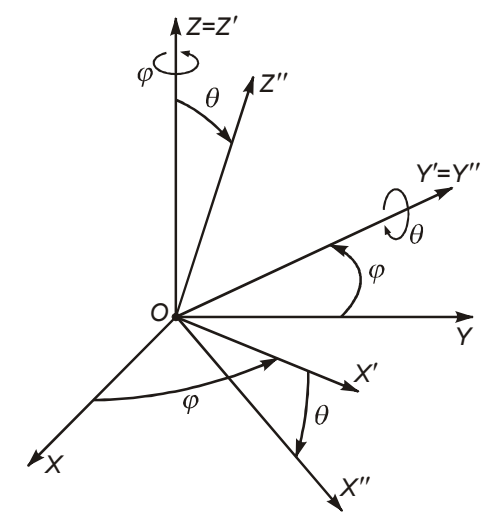

Fig. 1. Transformations $\quad X Y Z \rightarrow X^{\prime} Y^{\prime} Z^{\prime} \rightarrow X^{\prime \prime} Y^{\prime \prime} Z^{\prime \prime} \quad$ of Cartesian coordinate system made using successive rotations by angle $\varphi$ around $Z$ axis and by angle $\theta$ around $Y^{\prime}$ axis.

Transformations of the coordinate system under rotations around the principal axes $X, Y$ and $Z$ are described by so-called transformation matrices $\mathbf{T}_{x}(\chi), \mathbf{T}_{y}(\theta)$ and $\mathbf{T}_{z}(\varphi)$ :

$$
\mathbf{T}_{x}(\chi)=\left[\begin{array}{ccc}
1 & 0 & 0 \\
0 & \cos \chi & \sin \chi \\
0 & -\sin \chi & \cos \chi
\end{array}\right], \mathbf{T}_{y}(\theta)=\left[\begin{array}{ccc}
\cos \theta & 0 & -\sin \theta \\
0 & 1 & 0 \\
\sin \theta & 0 & \cos \theta
\end{array}\right], \mathbf{T}_{z}(\varphi)=\left[\begin{array}{ccc}
\cos \varphi & \sin \varphi & 0 \\
-\sin \varphi & \cos \varphi & 0 \\
0 & 0 & 1
\end{array}\right] .
$$

In our case we deal with sequential rotations around the $Z$ axis by the angle $+\varphi$ and around the $Y^{\prime}$ axis by the angle $+\theta$. Then the resulting transformation matrix $\mathbf{T} 2(\varphi, \theta)$ is obtained by multiplying the elementary matrices $\mathbf{T}_{y}(\theta)$ and $\mathbf{T}_{z}(\varphi)$ : 


$$
\mathbf{T 2}(\varphi, \theta)=\mathbf{T}_{y}(\theta) \times \mathbf{T}_{z}(\varphi)=\left[\begin{array}{ccc}
\cos \theta \cos \varphi & \cos \theta \sin \varphi & -\sin \theta \\
-\sin \varphi & \cos \varphi & 0 \\
\sin \theta \cos \varphi & \sin \theta \sin \varphi & \cos \theta
\end{array}\right] .
$$

Let us assume that the AW propagates along the $Z^{\prime \prime}$ axis. This means that the AW vector (AWV) $\mathbf{K}$ and the unit vector of wavefront normal (VWN) $\xi=\mathbf{K} /|\mathbf{K}|(|\xi|=1)$ are parallel to the $Z^{\prime \prime}$ axis: $\mathbf{K} \| Z^{\prime \prime}$ and $\xi \| Z^{\prime \prime}$. It is seen from Fig. 1 that the angles $\varphi$ and $\theta$ are respectively azimuthal angle and polar (or zenith, or inclination) angle of the VWN $\xi$ in the spherical coordinate system. The components of the VWN $\xi$ written in the $X Y Z$ system are nothing but directional cosines $\alpha, \beta$ and $\gamma$ of the AWV K:

$$
\begin{array}{ll}
\alpha=\xi_{x}=\xi_{1}=\sin \theta \cos \varphi, & \beta=\xi_{y}=\xi_{2}=\sin \theta \sin \varphi, \\
\gamma=\xi_{z}=\xi_{3}=\cos \theta, & \xi=[\sin \theta \cos \varphi ; \sin \theta \sin \varphi ; \cos \theta]
\end{array}
$$

It is known that searching for plane-wave solutions of the three-dimensional equation of motion leads to the Christoffel equation which represents a basic equation of $\mathrm{AW}$ propagation theory. This equation is as follows:

$$
\Lambda \times \mathbf{u}=v^{2} \mathbf{u},
$$

where $\boldsymbol{\Lambda}$ denotes a so-called reduced Christoffel tensor (RCT), $\mathbf{u}$ the displacement vector, and $v$ the phase AW velocity. The RCT $\boldsymbol{\Lambda}$ is related to the conventional Christoffel tensor $\boldsymbol{\Gamma}$ as

$$
\Lambda=\frac{1}{\rho} \Gamma .
$$

The symmetric second-rank Christoffel tensor $\boldsymbol{\Gamma}$ is expressed through the four-rank elasticstiffness tensor $\mathbf{C}$ and the VWN $\xi$ :

$$
\Gamma=\xi \cdot \mathbf{C} \cdot \xi, \quad \text { or } \Gamma_{i l}=C_{i j k l} \xi_{j} \xi_{k} .
$$

Then the components $\lambda_{i l}$ of the RCT $\boldsymbol{\Lambda}$ can be written as

$$
\lambda_{i l}=\frac{1}{\rho} \sum_{j=1}^{3} \sum_{k=1}^{3} C_{i j k l} \xi_{j} \xi_{k} .
$$

The last relationship can be represented in expanded form as

$$
\left[\begin{array}{l}
\lambda_{11} \\
\lambda_{22} \\
\lambda_{33} \\
\lambda_{23} \\
\lambda_{13} \\
\lambda_{12}
\end{array}\right]=\frac{1}{\rho}\left[\begin{array}{cccccc}
C_{11} & C_{66} & C_{55} & 2 C_{56} & 2 C_{15} & 2 C_{16} \\
C_{66} & C_{22} & C_{44} & 2 C_{24} & 2 C_{46} & 2 C_{26} \\
C_{55} & C_{44} & C_{33} & 2 C_{34} & 2 C_{35} & 2 C_{45} \\
C_{16} & C_{26} & C_{45} & \left(C_{46}+C_{25}\right)\left(C_{14}+C_{56}\right)\left(C_{12}+C_{66}\right) \\
C_{15} & C_{46} & C_{35} & \left(C_{45}+C_{36}\right)\left(C_{13}+C_{55}\right)\left(C_{14}+C_{56}\right) \\
C_{56} & C_{24} & C_{34} & \left(C_{44}+C_{23}\right)\left(C_{36}+C_{45}\right)\left(C_{45}+C_{46}\right)
\end{array}\right] \times\left[\begin{array}{c}
\xi_{1}^{2} \\
\xi_{2}^{2} \\
\xi_{3}^{2} \\
\xi_{2} \xi_{3} \\
\xi_{1} \xi_{3} \\
\xi_{1} \xi_{2}
\end{array}\right] .
$$

Since the RCT $\Lambda$ is a symmetric second-rank tensor, its indicative surface is in general an ellipsoid. It represents a surface of AW slowness $s\left(s=v^{-1}\right)$. This can be described using an optical-indicatrix type equation, provided that the components $B_{i j}$ of the dielectric impermeability tensor are replaced by the components $\lambda_{i l}$ of the RCT: $B_{i j} \rightarrow \lambda_{i j}$. Accordingly, the principal refractive indices $n_{1}, n_{2}$ and $n_{3}$ correspond to the principal slownesses $s_{1}, s_{2}$ and $s_{3}: n_{1} \rightarrow s_{1}$, $n_{2} \rightarrow s_{2}$ and $n_{3} \rightarrow s_{3}$. Similar to the optical indicatrix, the central cross section of the indicative surface of the RCT by the plane perpendicular to the AW propagation direction (i.e., to the VWN, $\left.\xi \| Z^{\prime \prime}\right)$ is an ellipse. The principal axes of this ellipse give directions of the two axes of coordinate 'eigensystem' of the RCT $\Lambda$, which is inherent to a given AW propagation direction $\xi \| Z$ '. 
In order to find the orientation of the principal axes of the ellipse, one has to transform the RCT $\Lambda$ from the $X Y Z$ system into the $X^{\prime \prime} Y^{\prime \prime} Z^{\prime \prime}$ system, using the following rule:

$$
\boldsymbol{\Lambda}^{\prime \prime}=\mathbf{T} 2(\varphi, \theta) \times \mathbf{\Lambda} \times \mathbf{T} \mathbf{2}^{-1}(\varphi, \theta) .
$$

The ellipse searched for lies in the plane $X^{\prime \prime} Y^{\prime \prime}$ and, in general, its principal axes form some angle $\psi$ with the $X^{\prime \prime}$ and $Y^{\prime \prime}$ axes, which is given by

$$
\tan 2 \psi=\frac{2 \lambda_{12}^{\prime \prime}}{\lambda_{22}^{\prime \prime}-\lambda_{11}^{\prime \prime}} .
$$

From Eq. (9) we obtain

$$
\left.\begin{array}{l}
\lambda_{11}^{\prime \prime}=\left(\lambda_{11} \cos ^{2} \varphi+\lambda_{22} \sin ^{2} \varphi+\lambda_{12} \sin 2 \varphi\right) \cos ^{2} \theta+\lambda_{33} \sin ^{2} \theta-\left(\lambda_{13} \cos \varphi+\lambda_{23} \sin \varphi\right) \sin 2 \theta, \\
\lambda_{22}^{\prime \prime}=\lambda_{11} \sin ^{2} \varphi+\lambda_{22} \cos ^{2} \varphi-\lambda_{12} \sin 2 \varphi, \\
\lambda_{12}^{\prime \prime}=\left[\frac{1}{2}\left(\lambda_{22}-\lambda_{11}\right) \sin 2 \varphi+\lambda_{12} \cos 2 \varphi\right] \cos \theta+\left(\lambda_{13} \sin \varphi-\lambda_{23} \cos \varphi\right) \sin \theta .
\end{array}\right\}
$$

Substituting Eqs. (11) into Eq. (10), we find the angle $\psi$ :

$$
\psi=\frac{1}{2} \arctan \left\{\frac{\left[\left(\lambda_{22}-\lambda_{11}\right) \sin 2 \varphi+2 \lambda_{12} \cos 2 \varphi\right] \cos \theta+2\left(\lambda_{13} \sin \varphi-\lambda_{23} \cos \varphi\right) \sin \theta}{\left[\begin{array}{l}
\lambda_{11}\left(\sin ^{2} \varphi-\cos ^{2} \theta \cos ^{2} \varphi\right)+\lambda_{22}\left(\cos ^{2} \varphi-\cos ^{2} \theta \sin ^{2} \varphi\right)+ \\
+\left(\lambda_{33}-\lambda_{12} \sin 2 \varphi\right) \sin ^{2} \theta-\left(\lambda_{13} \cos \varphi+\lambda_{23} \sin \varphi\right) \sin 2 \theta
\end{array}\right]}\right\} .
$$

After rotating the coordinate system $X^{\prime \prime} Y^{\prime \prime} Z^{\prime \prime}$ around the $Z^{\prime \prime}$ axis by the angle $\psi$, we obtain a new system $X^{\prime \prime \prime} Y^{\prime \prime \prime} Z^{\prime \prime}$, of which $X^{\prime \prime \prime}$ and $Y^{\prime \prime \prime}$ axes are directed along the principal axes of the ellipse, while the axis $Z^{\prime \prime \prime}=Z^{\prime \prime}$ is parallel to the VWN $\xi$. The matrix of transformation $X Y Z \rightarrow X^{\prime \prime \prime} Y^{\prime \prime \prime} Z^{\prime \prime \prime}$ looks like

$$
\begin{aligned}
& \mathbf{T 3}(\varphi, \theta, \psi)=\mathbf{T}_{z}(\psi) \times \mathbf{T} 2(\varphi, \theta)=\mathbf{T}_{z}(\psi) \times \mathbf{T}_{y}(\theta) \times \mathbf{T}_{z}(\varphi)= \\
& =\left[\begin{array}{ccc}
(\cos \theta \cos \varphi \cos \psi-\sin \varphi \sin \psi) & (\cos \theta \sin \varphi \cos \psi+\cos \varphi \sin \psi) & -\sin \theta \cos \psi \\
(-\cos \theta \cos \varphi \sin \psi-\sin \varphi \cos \psi) & (-\cos \theta \sin \varphi \sin \psi+\cos \varphi \cos \psi) & \sin \theta \sin \psi \\
\sin \theta \cos \varphi & \sin \theta \sin \varphi & \cos \theta
\end{array}\right] .
\end{aligned}
$$

The coordinate system $X^{\prime \prime \prime} Y^{\prime \prime} Z$ '"' is a 'reference' system for representation of the strain tensor components, which are induced by each of the three acoustic modes propagating along the VWN $\xi$. One of the versors of the coordinate system is given by the VWN $\xi$, which is written in the initial system (see Eq. (3)). The two other versors $\mathbf{t} 1$ and $\mathbf{t} \mathbf{2}$ are obtained by inversely transforming $X^{\prime \prime \prime} Y^{\prime \prime \prime} Z^{\prime \prime \prime} \rightarrow X Y Z$ according to the formula

$$
\begin{aligned}
& \mathbf{t} 1=\mathbf{T 3}^{-1}(\varphi, \theta, \psi) \times\left[\begin{array}{l}
1 \\
0 \\
0
\end{array}\right]=\left[\begin{array}{c}
\cos \theta \cos \varphi \cos \psi-\sin \varphi \sin \psi \\
\cos \theta \sin \varphi \cos \psi+\cos \varphi \sin \psi \\
-\sin \theta \cos \psi
\end{array}\right], \\
& \mathbf{t 2}=\mathbf{T 3}^{-1}(\varphi, \theta, \psi) \times\left[\begin{array}{l}
0 \\
1 \\
0
\end{array}\right]=\left[\begin{array}{c}
-\cos \theta \cos \varphi \sin \psi-\sin \varphi \cos \psi \\
-\cos \theta \sin \varphi \sin \psi+\cos \varphi \cos \psi \\
\sin \theta \sin \psi
\end{array}\right] .
\end{aligned}
$$

Comparing Eqs. (3) with Eqs. (13)-(15), one can see that the transformation matrices $\mathbf{T} 3(\varphi, \theta, \psi)$ and $\mathbf{T} 3^{-1}(\varphi, \theta, \psi)$ are defined by the components of $\boldsymbol{\xi}$, $\mathbf{t} \mathbf{1}$ and $\mathbf{t} \mathbf{2}$ versors:

$$
\mathbf{T 3}(\varphi, \theta, \psi)=\left[\begin{array}{ccc}
t 1_{1} & t 1_{2} & t 1_{3} \\
t 2_{1} & t 2_{2} & t 2_{3} \\
\xi_{1} & \xi_{2} & \xi_{3}
\end{array}\right], \quad \mathbf{T 3}^{-1}(\varphi, \theta, \psi)=\left[\begin{array}{ccc}
t 1_{1} & t 2_{1} & \xi_{1} \\
t 1_{2} & t 2_{2} & \xi_{2} \\
t 1_{3} & t 3_{3} & \xi_{3}
\end{array}\right] .
$$


As seen from the Christoffel equation, the eigenvalues of the RCT $\boldsymbol{\Lambda}$ are squared velocities of the acoustic eigenwaves $\left(\lambda_{e 1}=v_{1}^{2}, \lambda_{e 2}=v_{2}^{2}\right.$ and $\left.\lambda_{e 3}=v_{3}^{2}\right)$, whereas their displacement vectors $\mathbf{u 1}$, $\mathbf{u} 2$ and $\mathbf{u 3}$ are eigenvectors of the RCT $\boldsymbol{\Lambda}$. Moreover, the length of the latter vectors is unit: $|\mathbf{u} \mathbf{1}|=|\mathbf{u} \mathbf{2}|=|\mathbf{u} \mathbf{3}|=1$. Since the RCT $\mathbf{\Lambda}$ is written in the initial $X Y Z$ system, its eigenvectors $\mathbf{u 1 ,} \mathbf{u} 2$ and $\mathbf{u} \mathbf{3}$ are also expressed in this system. To rewrite them in the 'reference' coordinate system $X^{\prime \prime \prime} Y^{\prime \prime \prime} Z^{\prime \prime \prime}$, the following relations can be used:

$$
\begin{aligned}
& u 1_{1}^{\prime \prime \prime}=\mathbf{u 1} \cdot \mathbf{t 1}, \quad u 1_{2}^{\prime \prime \prime}=\mathbf{u 1} \cdot \mathbf{t} 2, \quad u 1_{3}^{\prime \prime \prime}=\mathbf{u 1} \cdot \xi \\
& \left.u 2_{1}^{\prime \prime \prime}=\mathbf{u} \mathbf{2} \cdot \mathbf{t} 1, u 2_{2}^{\prime \prime \prime}=\mathbf{u} \mathbf{2} \cdot \mathbf{t} \mathbf{2}, u 2_{3}^{\prime \prime \prime}=\mathbf{u} \mathbf{2} \cdot \xi ;\right\} \text {. } \\
& u 3_{1}^{\prime \prime \prime}=\mathbf{u} \mathbf{3} \cdot \mathbf{t} 1, u 3_{2}^{\prime \prime \prime}=\mathbf{u} \mathbf{3} \cdot \mathbf{t} \mathbf{2}, u 3_{3}^{\prime \prime \prime}=\mathbf{u} \mathbf{3} \cdot \xi ;
\end{aligned}
$$

It is known from Ref. [2] that the strain tensor components $e_{i j}$ are expressed in terms of coordinate derivatives of the displacement vectors:

$$
e_{i j}=\frac{1}{2}\left(\frac{d u_{i}}{d x_{j}}+\frac{d u_{j}}{d x_{i}}\right) .
$$

Since the plane AW propagates in the direction $\xi \| Z^{\prime \prime \prime}$, the displacement of particles in a crystal depends only on the coordinate $Z^{\prime \prime \prime}$ (see Fig. 2). Then the derivatives of the displacement vectors concerned with $X^{\prime \prime \prime}$ and $Y^{\prime \prime \prime}$ are equal to zero. As a result, we obtain the following normalized components of the strain tensor for the three acoustic eigenwaves:

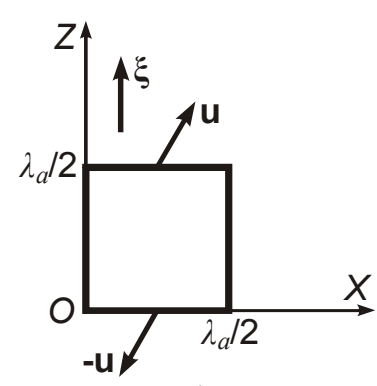

a)

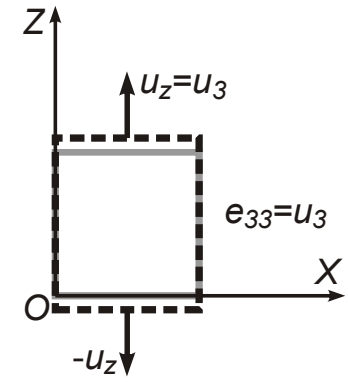

b)

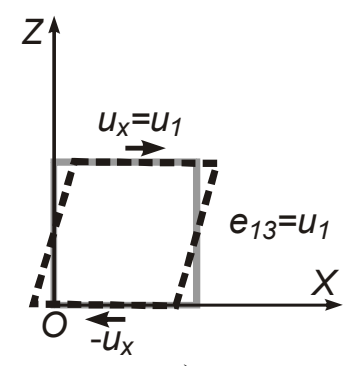

c)

Fig. 2. Deformations of elementary crystal volume appearing when the $A W$ with the $W W N \xi \| Z$ and the displacement vector $\mathbf{u}$ propagates in crystal, and appearance of normal $\left(e_{33}\right)$ and shear $\left(e_{13}\right)$ components of the strain tensor. The both vectors $\xi$ and $\mathbf{u}$ lie in $X Z$ plane.

$$
\left.\begin{array}{l}
e 1_{11}^{\prime \prime \prime}=0 ; \quad e 1_{22}^{\prime \prime \prime}=0 ; \quad e 1_{33}^{\prime \prime \prime}=u 1_{3}^{\prime \prime \prime} ; \quad e 1_{12}^{\prime \prime \prime}=0 ; \quad e 1_{13}^{\prime \prime \prime}=u 1_{1}^{\prime \prime \prime} ; e 1_{23}^{\prime \prime \prime}=u 1_{2}^{\prime \prime \prime}, \\
e 2_{11}^{\prime \prime \prime}=0 ; \quad e 2_{22}^{\prime \prime \prime}=0 ; \quad e 2_{33}^{\prime \prime \prime}=u 2_{3}^{\prime \prime \prime} ; \quad e 2_{12}^{\prime \prime \prime}=0 ; \quad e 2_{13}^{\prime \prime \prime}=u 2_{1}^{\prime \prime \prime} ; e 2_{23}^{\prime \prime \prime}=u 2_{2}^{\prime \prime \prime}, \\
e 3_{11}^{\prime \prime \prime}=0 ; \quad e 3_{22}^{\prime \prime \prime}=0 ; \quad e 3_{33}^{\prime \prime \prime}=u 3_{3}^{\prime \prime \prime} ; \quad e 3_{12}^{\prime \prime \prime}=0 ; \quad e 3_{13}^{\prime \prime \prime}=u 3_{1}^{\prime \prime \prime} \quad e 3_{23}^{\prime \prime \prime}=u 3_{2}^{\prime \prime \prime} .
\end{array}\right\}
$$

For each of the eigenwaves, there are three nonzero components of the strain tensor in the 'reference' coordinate system $X^{\prime \prime \prime} Y^{\prime \prime \prime} Z$ '":

$$
e_{i 13}^{\prime \prime \prime}=\mathbf{u}_{i} \cdot \mathbf{t} 1, \quad e_{i 23}^{\prime \prime \prime}=\mathbf{u}_{i} \cdot \mathbf{t} 2, \quad e_{i 33}^{\prime \prime \prime}=\mathbf{u}_{i} \cdot \xi .
$$

Taking Eqs. (17) and Eqs. (2) into account, one can write out the strain tensors $\mathbf{E}_{i}^{\prime \prime \prime}(i=1,2,3)$ for the three acoustic eigenmodes that propagate along the VWN $\xi$ :

$$
\mathbf{E}_{i}^{\prime \prime \prime}=\left[\begin{array}{ccc}
0 & 0 & e_{i 13}^{\prime \prime \prime} \\
0 & 0 & e_{i 23}^{\prime \prime \prime} \\
e_{i 13}^{\prime \prime \prime} & e_{i 23}^{\prime \prime \prime} & e_{i 33}^{\prime \prime \prime}
\end{array}\right]=\left[\begin{array}{ccc}
0 & 0 & \left(\mathbf{u}_{i} \cdot \mathbf{t} 1\right) \\
0 & 0 & \left(\mathbf{u}_{i} \cdot \mathbf{t} 2\right) \\
\left(\mathbf{u}_{i} \cdot \mathbf{t 1}\right) & \left(\mathbf{u}_{i} \cdot \mathbf{t} 2\right) & \left(\mathbf{u}_{i} \cdot \xi\right)
\end{array}\right] .
$$


This tensor can be transformed to the initial coordinate system as follows:

$$
\mathbf{E}_{i}=\mathbf{T 3}^{-1}(\varphi, \theta, \psi) \times \mathbf{E}_{i}^{\prime \prime \prime} \times \mathbf{T} 3(\varphi, \theta, \psi) .
$$

Substituting Eqs. (13) and (21) into Eq. (22), we obtain all the components of the strain tensors induced by the three acoustic eigenwaves propagating along the VWN $\xi=\{\sin \theta \cos \varphi$; $\sin \theta \sin \varphi ; \cos \theta\}$. In the initial coordinate system they are given by the relations

$$
\left.\begin{array}{rl}
e_{i 11}= & \left(e_{i 13}^{\prime \prime \prime} \cos \psi-e_{i 23}^{\prime \prime \prime} \sin \psi\right) \sin 2 \theta \cos ^{2} \varphi \\
& -\left(e_{i 13}^{\prime \prime \prime} \sin \psi+e_{i 23}^{\prime \prime} \cos \psi\right) \sin \theta \sin 2 \varphi+e_{i 33}^{\prime \prime \prime} \sin ^{2} \theta \cos ^{2} \varphi ; \\
e_{i 12}= & \frac{1}{2}\left(e_{i 13}^{\prime \prime \prime} \cos \psi-e_{i 23}^{\prime \prime \prime} \sin \psi\right) \sin 2 \theta \sin 2 \varphi \\
& +\left(e_{i 13}^{\prime \prime \prime} \sin \psi+e_{i 23}^{\prime \prime \prime} \cos \psi\right) \sin \theta \cos 2 \varphi+\frac{1}{2} e_{i 33}^{\prime \prime \prime} \sin ^{2} \theta \sin 2 \varphi ; \\
e_{i 13}= & \left(e_{i 13}^{\prime \prime \prime} \cos \psi-e_{i 23}^{\prime \prime \prime} \sin \psi\right) \cos 2 \theta \cos \varphi \\
& -\left(e_{i 13}^{\prime \prime \prime} \sin \psi+e_{i 23}^{\prime \prime \prime} \cos \psi\right) \cos \theta \sin \varphi+\frac{1}{2} e_{i 33}^{\prime \prime \prime} \sin 2 \theta \cos \varphi ; \\
e_{i 22}= & \left(e_{i 13}^{\prime \prime \prime} \cos \psi-e_{i 23}^{\prime \prime 2} \sin \psi\right) \sin 2 \theta \sin ^{2} \varphi \\
& +\left(e_{i 13}^{\prime \prime \prime} \sin \psi+e_{i 23}^{\prime \prime \prime} \cos \psi\right) \sin \theta \sin 2 \varphi+e_{i 33}^{\prime \prime \prime} \sin ^{2} \theta \sin ^{2} \varphi ; \\
e_{i 23}= & \left(e_{i 13}^{\prime \prime \prime} \cos \psi-e_{i 23}^{\prime \prime \prime} \sin \psi\right) \cos 2 \theta \sin \varphi \\
& +\left(e_{i 13}^{\prime \prime \prime} \sin \psi+e_{i 23}^{\prime \prime \prime} \cos \psi\right) \cos \theta \cos \varphi+\frac{1}{2} e_{i 33}^{\prime \prime \prime} \sin 2 \theta \sin \varphi ; \\
e_{i 33} & =e_{i 33}^{\prime \prime \prime} \cos { }^{2} \theta-\left(e_{i 13}^{\prime \prime \prime} \cos \psi-e_{i 23}^{\prime \prime \prime} \sin \psi\right) \sin 2 \theta .
\end{array}\right\}
$$

Finally, multiplying these tensors by the tensor of strain-optical coefficients peculiar for a crystal under study, one can derive the induced increments of all the components of the dielectric impermeability tensor. Then, the AO-induced increments of the refractive indices can be obtained. In this manner, the EEC for a given $\mathrm{AO}$ interaction geometry can be calculated.

\section{Conclusion}

In the present work we have derived analytical relations for the strain tensor components, which are caused by AWs with arbitrary wave vector directions in crystals. The first technique used for obtaining these relations is the standard rotation-matrix approach. The second technique is constructing cross sections of the indicative surfaces that describe the Christoffel tensor. These cross sections are made by the planes perpendicular to the wave vectors. The relations obtained by us can be used for the consistent analysis of AOFM anisotropy.

\section{Acknowledgement.}

The authors acknowledge financial support of the present study from the Ministry of Education and Science of Ukraine (the Projects \# 0117 U000802 and 0118U003999).

\section{References}

1. Balakshyi V I, Paryhyn V N and Chyrkov L E. Physical foundations of acoustooptics. Moscow: Radio and Communications (1985).

2. Sirotin Yu I and Shaskolskaya M P. Fundamentals of crystal physics. Moscow: Mir, 1982.

3. Buryy O, Andrushchak N, Ratych A, Demyanyshyn N, Mytsyk B and Andrushchak A, 2017. Global maxima for the acoustooptic effect in $\mathrm{SrB}_{4} \mathrm{O}_{7}$ crystals. Appl. Opt. 56: 1839-1845.

4. Andrushchak A S, Chernyhivsky E M, Gotra Z Yu, Kaidan M V, Kityk A V, Andrushchak N 
A, Maksymyuk T A, Mytsyk B G and Schranz W, 2010. Spatial anisotropy of the acoustooptical efficiency in lithium niobate crystals. J. Appl. Phys. 108: 103118.

5. Zyuryukin Yu A, Zavarin S V and Yulaev A N, 2009. Characteristic features of wideband anisotropic light diffraction in lithium-niobate crystal by a longitudinal acoustic wave. Opt. Spectrosc. 107: 152-156.

6. Fujii $\mathrm{Y}$ and Hayashi H, 1977. Acousto-optic tunable filter using $\mathrm{LiNbO}_{3}$ crystals. Proc. SPIE. 99: 110-115.

7. Mys O, Krupych O and Vlokh R, 2018. Anisotropy of acousto-optic figure of merit in lithium tetraborate crystals. J. Mod. Opt. 65: 1486-1494.

8. Mys O, Kostyrko M, Krupych O and Vlokh R, 2015. Anisotropy of the acousto-optic figure of merit for $\mathrm{LiNbO}_{3}$ crystals: isotropic diffraction. Appl. Opt. 54: 8176-8186.

9. Mys O, Krupych $\mathrm{O}$ and Vlokh R, 2016. Anisotropy of an acousto-optic figure of merit for $\mathrm{NaBi}\left(\mathrm{MoO}_{4}\right)_{2}$ crystals. Appl. Opt. 55: 7941-7955.

10. Mys O, Adamenko D, Krupych O and Vlokh R, 2018. Effect of deviation from purely transverse and longitudinal polarization states of acoustic waves on the anisotropy of acoustooptic figure of merit: The case of $\mathrm{Tl}_{3} \mathrm{AsS}_{4}$ crystals. Appl. Opt. 57: 8320-8330.

Krupych O., Adamenko D. and Vlokh R. 2019. Analytical relations for the strain tensor components caused by acoustic waves with arbitrary wave vector directions in crystals: acoustooptic applications. Ukr.J.Phys.Opt. 20: 16 - 22. doi: 10.3116/16091833/20/1/16/2019

Анотація. Одержано аналітичні співвідношення для компонент тензора деформацій, спричинених акустичними хвилями з довільними напрямками хвильового вектора в кристалах. Підхід базується на стандартних поворотних матричях $і$ поперечних перерізах індикативних поверхонь тензора Крістоффеля, зроблених площинами, перпендикулярними до хвильового вектора. 